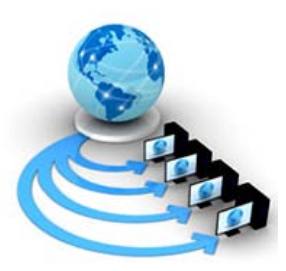

Volume 8, No. 9, November-December 2017

International Journal of Advanced Research in Computer Science

RESEARCH PAPER

\author{
Available Online at www.ijarcs.info
}

\title{
A REVIEW ON IMAGE SEGMENTATION TECHNIQUES WITH AN APPLICATION PERSPECTIVE
}

\author{
Kinjal Munot, Nishi Mehta, Sakshi Mishra \\ Student \\ Mukesh Patel School of Technology Management and \\ Engineering \\ Mumbai, Maharashtra, India
}

\author{
Ratnesh N. Chaturvedi \\ Asst. Professor \\ Mukesh Patel School of Technology Management \\ andEngineering \\ Mumbai, Maharashtra, India
}

\begin{abstract}
Image Segmentation is an important part of Image Processing. The first step in Image Analysis and Pattern Recognition is Image Segmentation. The process of Image Segmentation refers to thepartition of an image into different regions to gain information about the objects in the image based on the homogeneity or discontinuity of certain characteristics like color, intensity or texture. The purpose of this paper is to provide an overview of various image segmentation methods and their implementation in different color spaces. Their relative efficiency, advantages, and the problems they encounter are discussed. In order to understand how these methods are used, someapplications like XRadiography, Foreground detection in MRI and Facial feature detection are explained. It is observed that no image segmentation technique and algorithm work well with all types of images but these techniques work well specific to certain applications. Hence, it is critical to decide which method is the most suitable for a particular application based on its efficiency in that domain.
\end{abstract}

Keywords: Image Segmentation, Thresholding, Edge and Region Detection, Image Segmentation Applications.

\section{INTRODUCTION}

Image segmentation is the process of partitioning a digital image into multiple segments. It is a part of the Image Processing domain.It is very important to study the data of a processed image. One can identify the objects in an image by simply looking at it but it is difficult to be done by a computer algorithm.

The process of Image Segmentation is described in three stages: [1]

1)Image processing- removal of useless information from the image.

2)Initial object discrimination- objects are separated into groups with similar attributes.

3)Object boundary clean up- object boundaries are reduced to single pixel widths.

A good segmentation result shows characteristic such as coincident and non-coincident segmented pixels on region and edges, preservation of area, perimeter, and degree of curvature of the segmented boundary, statistics, entropy, center of mass and topology of the segmented regions.

Image Segmentation is based on two basic properties of the pixels in relation to their positions: Discontinuity and Regularity.

In this paper, various segmentation techniques are reviewed and how they are implemented in different color spaces as follows: section II discusses the technique and principle of Thresholding. Section III describes EdgeBased Segmentation and Section IV describes RegionBased Segmentation. Section V discusses superimposition of two regions in an image. Section VI discusses the applications of Thresholding and Edge Detection in XRadiography Images, Foreground Detection in MRI and
Detection of Facial features like Lips, Eyes, etc. Section VI describes edge detection using histogram color segmentation using HSV color space. In section VIII, the conclusion is presented where the purpose of this paper is summarized.

\section{THRESHOLDING}

Thresholding is a very important method of image segmentation. It is done by partitioning the pixels of the input image into two (binary) or more (multiple thresholding) values by comparison of the pixel values with a predefined threshold value independently [2].

Results of Thresholding can have the following issues like:

- The segmented area could be either larger or smaller than the image.

- $\quad$ Edges might not be attached.

- Under-segmentation

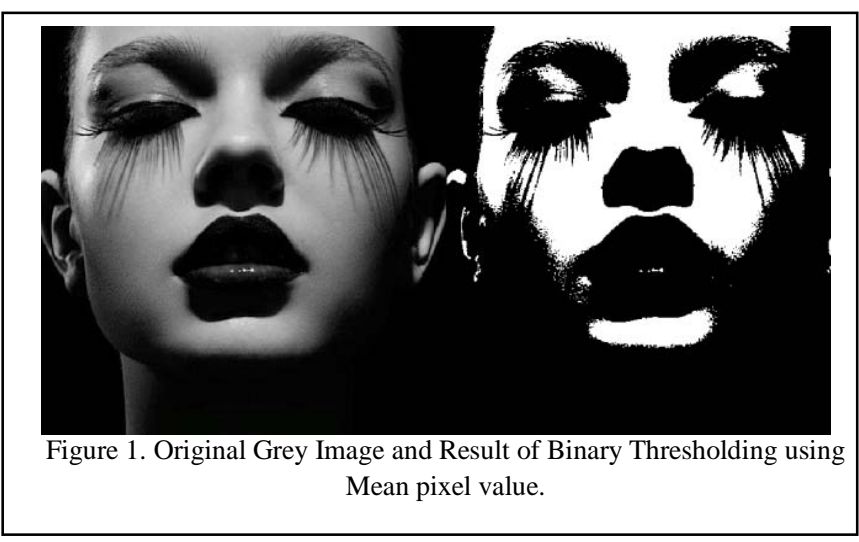

Figure 1 is an example of Binary Thresholding on a grey image.Thresholding is used to distinguish foreground from the background [3]. Binary thresholding is a common method of converting a grey-level image into a binary image 
by selecting a threshold value, below which all pixel values are classified as black (0) and the ones above this value as white (256).

P-tile method, Iterative threshold selection, Adaptive thresholding, Variable thresholding and, Mode methods are described in [3]. The algorithm to determine the most appropriate threshold value is yet to be found.

\section{EDGE BASED SEGMENTATION}

An edge is defined as a set of connected pixels that lie on the border of two regions with different grey values. Edge-based segmentation works on the pixel positions in the image that are parallel to the boundaries of the objects seen in the image.

The process of Edge Detection has three main steps which are Filtering, Differentiation and Localization.

Edge-Based Segmentation methods are classified as follows in Figure 2:

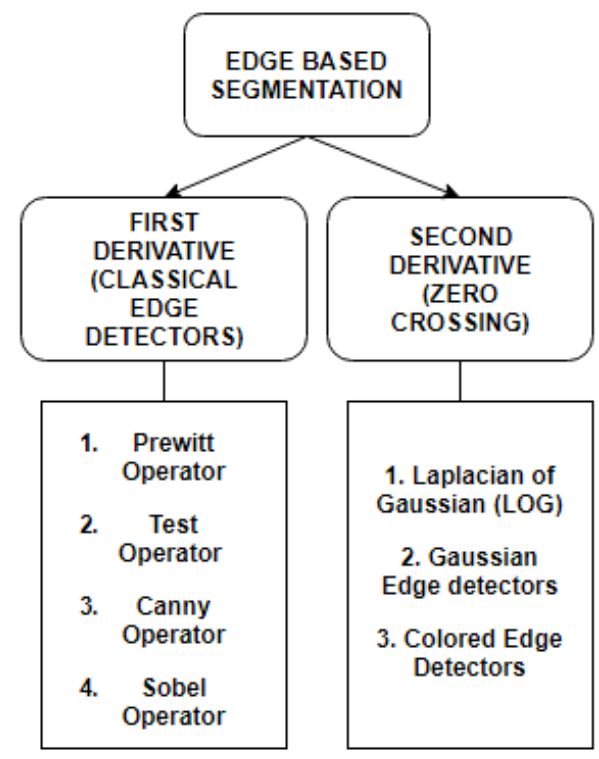

Figure 2. Classification of Edge Based Segmentation

The Canny Edge detector is described as a standard benchmark against which other edge detection algorithms are compared [4].

It involves four steps:

1. Smoothing by Gaussian convolution

2. Finding edge strength and edge directions

3. Non-maximal suppression

4. Hysteresis

It uses two thresholds: high and low. Any pixel above the high threshold is automatically considered an edge. Any pixel between the high and low threshold that is adjacent to an edge pixel is also considered an edge.

The disadvantage of this method is that it finds pixels near the edges but is not able to find exact edges.

Edge-Based segmented images have complications if the images are edge-less, noisy or textured.

\section{REGION-BASEDSEGMENTATION}

Region-Based methods are pixel-based methods that depict the splitting of an image into standardized areas of connected pixels through the application of homogeneity criteria among applicant groups of pixels. In Region Growing Algorithm the regions are grown up from the seed points to adjacent points depending on a region membership condition (pixel strength, grey level, or color) [2].

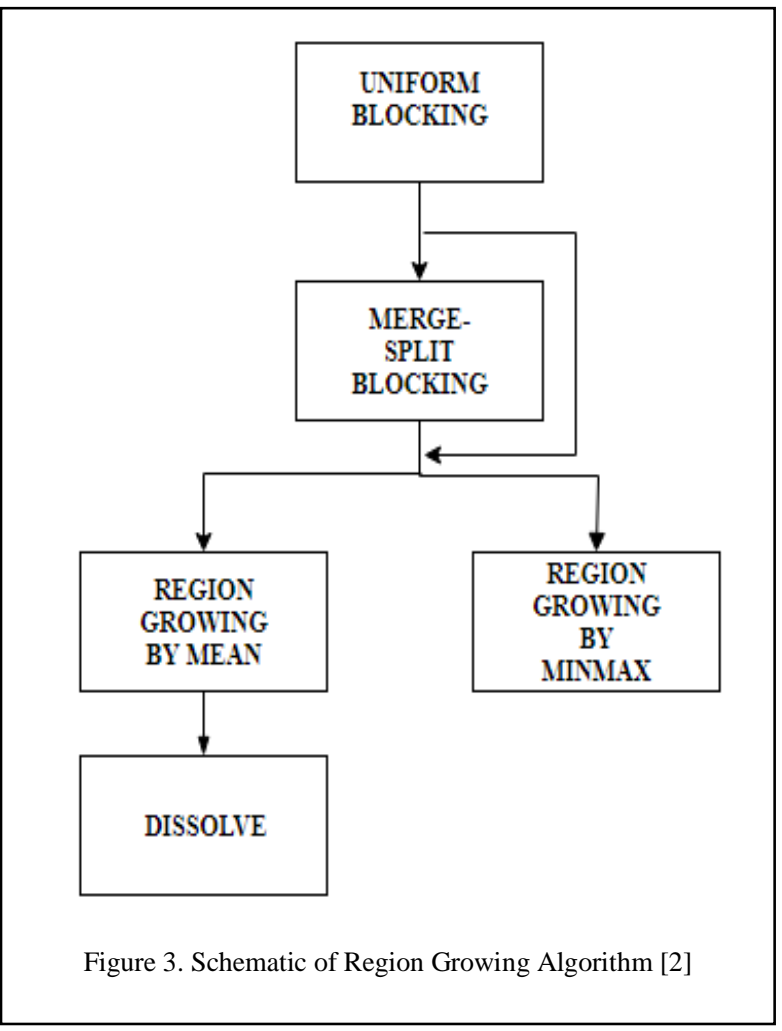

Figure 3 shows the schematic hierarchical classification of region growing.

In Region Based methods, the selection of appropriate seed points is difficult.

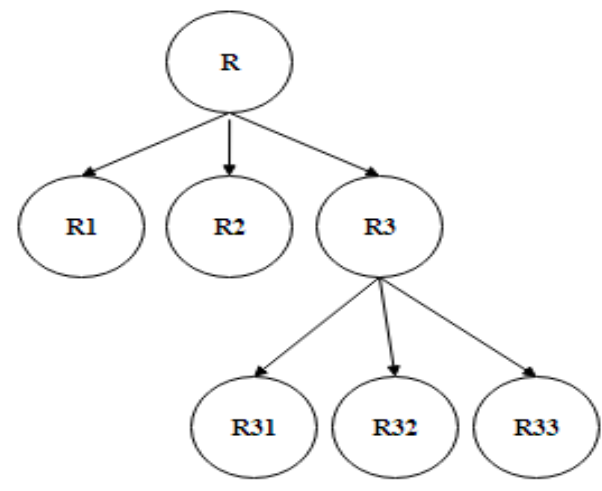

Figure 4. Quad Tree [3] 
Region Merge-Split Blocking is described in [3].Region Merging and Splitting involves breaking the image into quadrants. For this, a quadtree with root as the main image and child as subdivided regions are used as shown in Figure 4. Then, merging of any adjacent regions that are similar enough is done. This entire process is repeated till no more splitting takes place.

\section{SUPERIMPOSITION OF TWO REGIONS [5]}

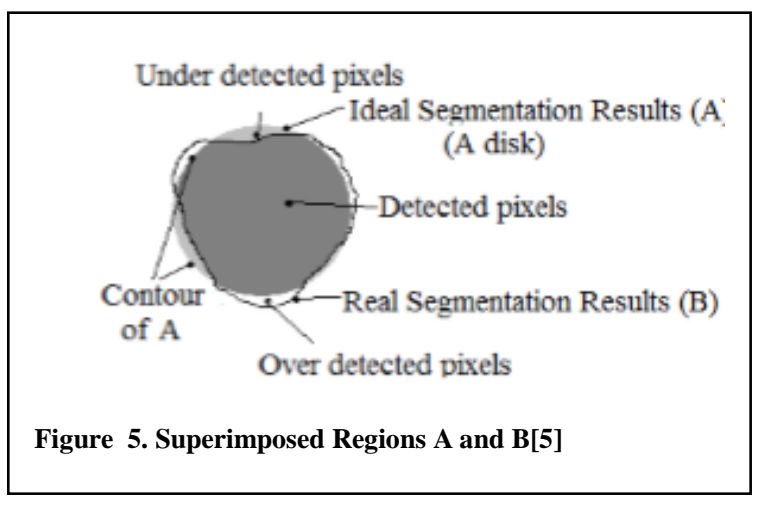

Let $\mathrm{A}$ represent the ideal segmentation result region and $\mathrm{B}$ represent the result in real segmentation map as shown in Figure 5. Here A and B are shown in a common support.

The discrepancy that exists is induced by the under detected and the over detected pixels. It could modify the overall shape of the resulting region, relocate it or even rotate it. [5] The six measures that look after the aspects of the missing segmented pixels are as follows:

- Under Detection and Over Detection Measures

- $\quad$ Shift Measure S
- $\quad$ Rotation Measure R

- Connectivity Measure Cn

- $\quad$ Compactness Measure CP

- $\quad$ Pixel Error Rates FP Rate and FN Rate

It is efficient to put together all the six measures and form a composite measure called CM for evaluation and comparison of region-based segmentation result.

\section{APPLICATIONS}

Practical application of Image Segmentation ranges from filtering of noisy images, medical applications like locating tumors and measuring tissue volumes, to locating objects in satellite images (roads, forests, etc.), Face Recognition and Fingerprint Recognition, etc.

\section{A. X-Radiography [6]}

Thresholding is applied on X-Radiography images that have two different background contrast levels. The idea is to split an image into regions of maximum homogeneity. Seeds are selected automatically by addressing both Otsu and Kittler's thresholds. [6]

Otsu and Kittler's methods are applied on X-radiography images in two phases:

Phase 1: Finding threshold values and identifying seed pixels.

Phase 2: Scanning the labeled pixels and applying functional criterion on it. Then, checking the neighboring pixels for at least 8-connected and summing them to the region if they are satisfying the criterion.

Figure 6 shows the comparison of segmentation results of Otsu's method, Kittler's method and the proposed method showing its efficiency

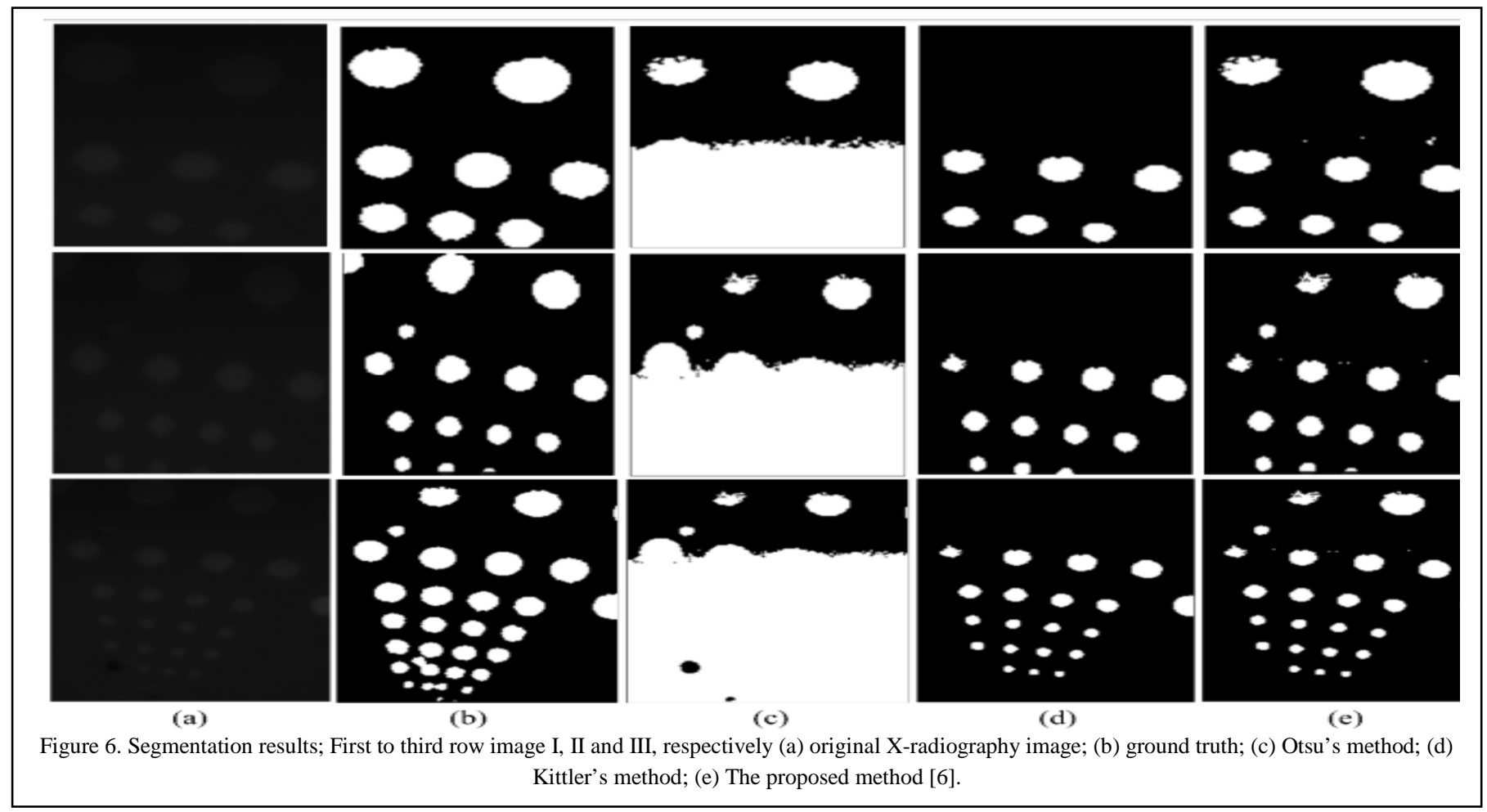




\section{B. Lip detection [7]}

An algorithm for lip detection in a single face imageusingbothRegion-based Segmentation and Edge Detection is proposed in [7].

Lip detection is an important area of research in image processing that has numerous applications. The proposed method is based on skin detection using acombination of HSV, YCbCr and normalized RGB color models; face detection using largest connected skin region and lip detection after lower part extraction by edge information. It can be applied to multiple types of images for lip detection but only those which contain a single face. There is a scope for improvement in the algorithm to detect eyes, ears, nose, etc.

Figure 7 shows the result of the proposed algorithm when implemented on a single face image.

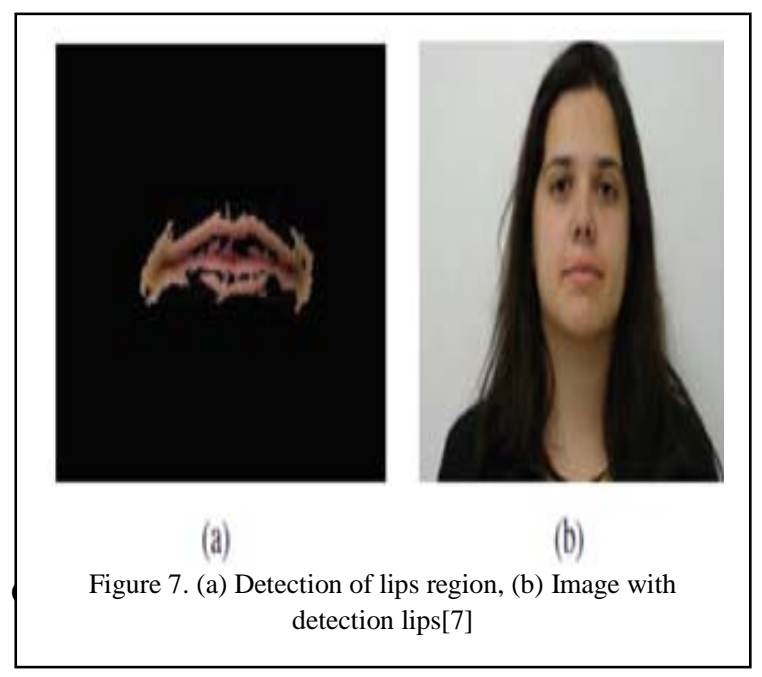

This algorithm discusses the detection and segmentationof face in an improved form. Initially, for theimage segmentation Voronoi diagram is used. This segmentation method may lead to a confusing result when the background intensities are similar to face intensities. To cope with such problem, distance transformation DisT is used to separate theface from its background. The function operates on the Euclidean distance between two given points (x1, y1) and (x2, y2), where

$$
\operatorname{Dis} T=\sqrt{\left(x_{1}-x_{2}\right)^{2}+\left(y_{1}-y_{2}\right)^{2}}
$$

To limit testing the whole image and for correct detection, these steps are carried out:

1. Ellipse fitting: For each connected component an ellipse is computed on the basis of its parameters which are thecenter, orientation, minor axis and major axis.

2. Aspect ratio: Face candidates are selected based on

$$
\forall \frac{a}{b} \leq \xi
$$

Where, $\boldsymbol{\xi}$ is a tolerance factor and a and b are the minor and major axis.
3. Euler number: The region with no holes is eliminated by computing the Euler number defined as:

4. $\mathrm{E}=\mathrm{C}-\mathrm{H}(3)$

Where $\mathrm{E}$ is the Euler number, $\mathrm{C}$ is the number of connected components, $\mathrm{H}$ is the number of holes.

The advantage of this method is that it does not depend on rotation or facial expression. But the existence of some features (facial hair, sunglasses, and occlusion) still a challenging task in face detection.

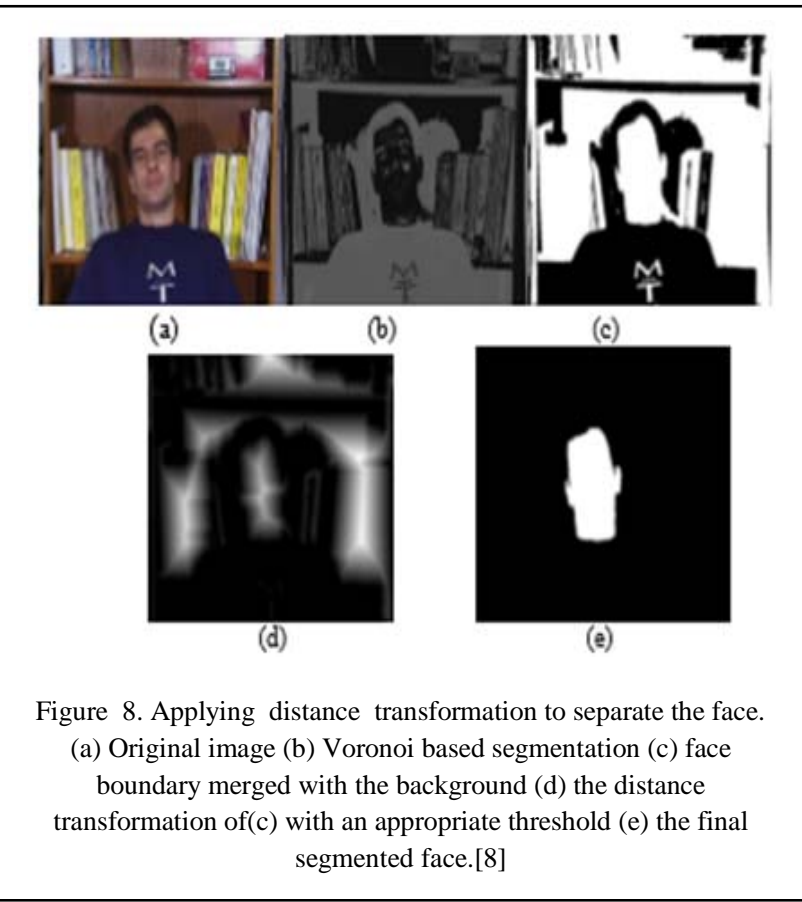

Figure 8 shows the result of the proposed face detection algorithm.

\section{Magnetic Resonance Image Segmentation [9]}

Every image can be separated into foreground and background or be grouped together based on various characteristics of the pixels.

Challenges faced in image segmentation are:Nonuniform illumination, noise, aninadequate model of theobject of interest, high dimensional data space, lots of information, many cues,clutter(cover) in natural scenes, occluded (close up) or overlapping objects. Hence, extracting the foreground image is important in image segmentation.

MRI Image Segmentation is an application of classifying foreground image. Algorithms such as anisotropic, diffusion and filtering are used and at second stage feature extraction and Sobel edge detection is done from the segmented result which is implemented by SVM classifier using MATLAB achieving the accuracy of $62.3 \%$. [9] 

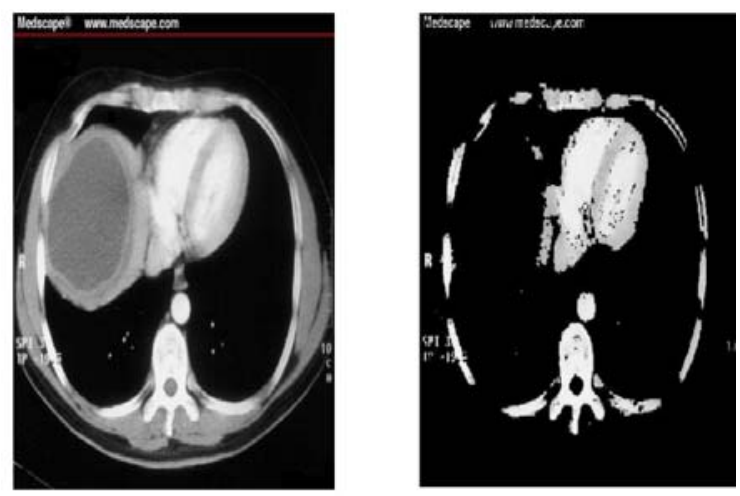

Figure 9: Original Image and Segmented Foreground Image [9]

This segmentation algorithm could be connected to other computer vision errands such as acknowledgment and recovery.

Figure 9 shows the segmented foreground of a MRI Image

\section{EDGE DETECTION AND HISTOGRAM COLOR SEGMENTATION [10]}

Till now only segmentation of grey or black and white images has been discussed.The paper [10] describes color image segmentation based on its Histogram. Color segmentation is the process of segmenting/separating features based on colors from a color image.Another way to do color segmentation is to convert the image color space into Hue Saturation Value (HSV) model. This way the color can be addressed only in ranges of hue and specified further by the saturation and value. In HSV color space, a histogram can separately represent the magnitude of hue, saturation, and value that corresponds to all pixels in an image. Hence, histogram color segmentation is applied to recognize significant colors.

Figure 10 represents the Hue, Saturation and Value Histogram of a color image.

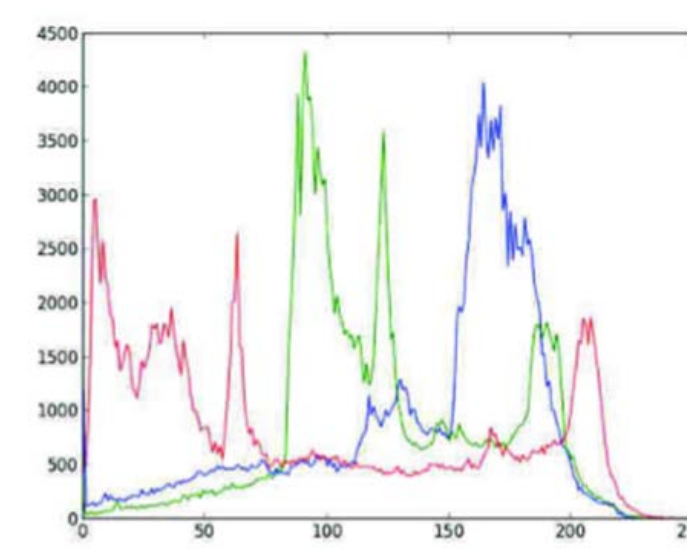

Figure 10.HSV Histogram [10]

\section{CONCLUSION}

The purpose of this paper was to discuss different popular image segmentation techniques and their application in different fields which is achieved. Fundamental properties and approaches of different techniques have been highlighted. The advantages and disadvantages of the methods have been highlighted. Even though there are numerous Image Segmentation methods available, each of them works on a specific concept. Hence, it is important to analyze which method is applicable to a particular domain.

With this analysis, it is concluded that there is no single algorithm that works well for all types of images, but specific algorithms can be applied to specific images in specific applications as observed in the Foreground Detection technique used in X-radiography images and in the Face Detection algorithm.

\section{REFERENCES}

[1] Ku. Vasundhara H. Lokhande, "Study of Region Base Segmentation Method",Volume 4, Issue 1, January 2014,in International Journal of Advanced Research in Computer Science and Software Engineering, pp.197-200

[2] Dr. K. Sathiyasekar, S. Karthick and A. Puraneeswari, "A Survey Based on Region Based Segmentation", Volume 7 Number, $3{ }^{\text {rd }}$ Jan 2014, International Journal of Engineering Trends and Technology (IJETT), pp.143-147

[3] M. Jogendra Kumar, Dr. GVS Raj Kumar, R. Vijay Kumar Reddy3,” REVIEW ON IMAGE SEGMENTATION TECHNIQUES” ,Volume 3, Issue 6, September 2014., International Journal of Scientific Research Engineering \& Technology (IJSRET), ISSN 2278 - 0882 ,pp.992-997

[4] HassanaGrema, "Region-Based Segmentation versus Edge Detection ", Fifth International Conference on Intelligent Information Hiding and Multimedia Signal Processing ,2009 ,pp.1217-1221

[5] AichaBayaGoumeidane, "Merging Discrepancy Measures for Region-Based SegmentationResults Evaluation and Comparison: Application to Thresholded Weld Defect RadiographicImages",8th International Symposium on Image and Signal Processing and Analysis (ISPA 2013),pp.78-82

[6] Purna Chandra Rao Bhagi, SaravananThangavel, Arunmuthu Krishnan, "Region growing based segmentation with automatic seed selection using threshold techniques on $\mathrm{X}$ radiography images 2016 IEEE International Conference on Computational Intelligence and Computing Research, 2016.

[7] Aleksandra Arsic, Milos Jordanski, and Milan Tuba, "Improved Lip Detection Algorithm Based on Region Segmentation and Edge Detection", $23^{\text {rd }}$ Telecommunications forum TELFOR, 2015, pp.472-475.

[8] Safa JIDA, Brahim AKSASSE, Mohammed OUANAN," Face segmentation and detection using Voronoi diagram and 2D histogram”, 978-1-5090-4062-9/17/\$31.00 (2017 IEEE.

[9] R.Kotteswari and K.G Sathiya "Analysis of Foreground Detection in MRI Images using Region Based segmentation”,International Conference on Communication and Signal Processing, April 6-8, 2016, India,pp. 1062-1065.

[10] Arnold Aribowo, GiorgyGunawan, Hendra Tjahyadi, "Adaptive Edge Detection and Histogram Color Segmentation for Centralized Vision of Soccer Robot", International Conference on Informatics and Computing (ICIC), 2016. 